\title{
Inappropriate Early Hypotension in Adolescents: A Form of Chronic Orthostatic Intolerance with Defective Dependent Vasoconstriction
}

\author{
JULIAN M. STEWART AND AMY WELDON \\ Departments of Pediatrics [J.M.S., A.W.] and Physiology [J.M.S.], The Center for Pediatric Hypotension,
} New York Medical College Valhalla, NY 10595, U.S.A.

\section{ABSTRACT}

\begin{abstract}
ABST
Instantaneous orthostatic hypotension (INOH) has been re-
ported in children and adolescents as a new entity of orthostatic
intolerance in children who underwent rapid standing as an
orthostatic stress test. Children with INOH were discovered
among patients presenting with symptoms of chronic orthostatic
intolerance, which is often related to orthostatic tachycardia. We
used head-up tilt table testing at $70^{\circ}$ to investigate children
presenting with symptoms of chronic orthostatic intolerance. We
compared 24 patients aged $12-17$ y, with chronic orthostatic
intolerance and symptoms for $\geq 3$ mo, with 13 healthy normal
control patients. We recorded continuous heart rate, blood pres-
sure, and respiratory rate and used venous occlusion strain gauge
plethysmography to measure calf and forearm blood flow while
supine and calf blood flow during head-up tilt. Patients with
chronic orthostatic intolerance fulfilled criteria for the postural
orthostatic tachycardia syndrome. Postural orthostatic tachycar-
dia syndrome patients were divided into two groups by the
occurrence of INOH. Supine forearm and calf arterial resistance
was decreased in patients with INOH ( $n=8$ ) compared with
postural orthostatic tachycardia syndrome patients without INOH
\end{abstract}
INOH has recently been reported as a new entity of orthostatic intolerance in children and adolescents who underwent rapid standing as an orthostatic stress test $(1,2)$. Data from that original study suggested that some patients developed systolic hypotension whereas others did not. Upright tachycardia was a common feature of all patients. Orthostatic tachycardia with COI has been described at least since 1940 (3) and more recently by investigators designating the syndrome POTS (for postural orthostatic tachycardia syndrome) $(4-6)$ or COI $(7,8)$ in adults. We and others have described the syndrome in children and adolescents $(6,9-12)$, often in the context of the

Received June 28, 2000; accepted October 12, 2000.

Correspondence and reprint requests: Julian M. Stewart, M.D., Ph.D., Department of Pediatrics, The Center for Pediatric Hypotension and The Division of Pediatric Cardiology, Suite 618, Munger Pavilion, New York Medical College, Valhalla, NY 10595, U.S.A.; e-mail: stewart@nymc.edu

Supported by 1RO3-AI45954 from the National Institutes of Health/National Institute of Allergy and Infectious Diseases. $(n=16)$ and compared with control $(n=13)$. Resting calf venous pressure was elevated, suggesting excess venous filling because of vasodilation. During early head-up tilt, calf blood flow increased markedly in $\mathrm{INOH}$, less in No-INOH, postural orthostatic tachycardia syndrome patients and least in control patients. Flow was temporally related to calf swelling and negatively correlated to hypotension. The data suggest that $\mathrm{INOH}$ occurs in patients with chronic orthostatic intolerance and orthostatic tachycardia and is related to rapid caudal blood flow when upright because of a vasoconstrictor defect. (Pediatr Res 50: 97-103, 2001)

$\quad$ Abbreviations
INOH, instantaneous orthostatic hypotension
COI, chronic orthostatic intolerance
POTS, postural orthostatic tachycardia syndrome
COI-POTS, patients with chronic orthostatic intolerance and
postural orthostatic tachycardia
HUT, head-up tilt
Pv, venous pressure

chronic fatigue syndrome (12). We suspect that vascular resistance is lower than healthy control subjects in many COIPOTS patients. We hypothesized that abnormally increased blood flow to the lower extremities in COI-POTS patients during orthostatic challenge related to reduced lower limb resistance causes a precipitous early fall in blood pressureergo INOH. To investigate this hypothesis we used venous occlusion plethysmography to study blood flow supine and during HUT, comparing 24 patients with COI-POTS with 13 healthy control patients.

\section{METHODS}

Patients and control subjects. We studied 24 patients aged 12-17 y (20 girls, 4 boys) referred to pediatric cardiology for COI. All patients had POTS on HUT associated with symptoms of orthostatic intolerance. Symptoms included lightheadedness, nausea and vomiting, palpitations, fatigue, headache, 
blurred vision, abnormal sweating, and a sensation of heat while upright with no other medical explanation for the symptoms. COI-POTS patients all complained of three or more symptoms of orthostatic intolerance for $\geq 3 \mathrm{mo}$. Ten children fulfilled the criteria for chronic fatigue syndrome as defined by the Centers for Disease Control and Prevention (13). No patient had previously fainted.

The control group comprised 13 healthy patients aged 11-18 y (10 girls, 3 boys). Healthy control patients were recruited from among adolescents referred for innocent heart murmur. Patients with a history of syncope or orthostatic intolerance were specifically excluded.

Only children found on cardiac examination to be free from structural or arrhythmic heart disease were eligible to participate. Enrolled patients were free of all obvious systemic illnesses and were not taking neurally active or vasoactive medications. Informed consent was obtained, and the Committee for the Protection of Human Subjects (IRB) of New York Medical College approved all protocols. Technically adequate tracings were obtained from all patients and control subjects.

Patient classification. Orthostatic tachycardia was determined from HUT results. Significant immediate orthostatic hypotension, defined as a $>25 \mathrm{~mm} \mathrm{Hg}$ fall in systolic blood pressure, was observed in eight patients. This is consistent with the consensus definition of the American Autonomic Society for orthostatic hypotension (14). POTS was diagnosed by symptoms of orthostatic intolerance during HUT associated with an increase in sinus heart rate of $>30$ beats per minute or to a rate of $>120$ beats per minute within the first 10 min of tilt. Hypotension was not requisite. Criteria were selected for consistency with the adult literature $(4,5)$. These may not be entirely appropriate to adolescents, in whom a higher heart rate may be more appropriate. Therefore, in our patients we required an increase in heart rate by $\geq 35$ beats per minute to define POTS.

Patient assessment. Laboratory tests included complete blood count with leukocyte differential; erythrocyte sedimentation rate; serum levels of alanine aminotransferase, total protein, albumin, globulin, alkaline phosphatase, calcium, phosphorus, glucose, blood urea nitrogen, electrolytes, and creatinine; determination of TSH; and urinalysis, which were normal in every instance. Patients were also tested for Lyme disease with Lyme ELISA and Western blot analysis because Lyme disease is endemic in our area. Patients with active Lyme disease were specifically excluded. Routine cardiovascular physical examinations were performed and were supplemented by ECG and echocardiographic assessments to rule out heart disease. All tests in study subjects were normal by study design (patients with any abnormalities were excluded).

Approximately 50 to $60 \%$ of all POTS patients give a history of antecedent infection.

Circulatory evaluation. Circulatory testing was performed in the laboratory during a single day. After overnight fast, tests began between $0900 \mathrm{~h}$ and $1000 \mathrm{~h}$. The ECG was monitored continuously. Blood pressure was continuously monitored with an arterial tonometer (Collin Instruments, San Antonio, TX, U.S.A.) placed on the right radial artery calibrated against oscillometric sphygmomanometer pressure. Tonometric arte- rial pressure was recalibrated every $5 \mathrm{~min}$. A respiratory impedance plethysmograph (Respitrace 200, NIMS Inc, Miami Beach, FL, U.S.A.) was used to monitor respiratory changes. Respiratory, ECG, and pressure data were interfaced to a personal computer through an A/D converter (DataQ Industries, Milwaukee, WI, U.S.A.), and custom software was used to produce, display, and store R-R intervals, respiratory rate, and blood pressure (mean, systolic, diastolic, and phasic tracings) on a continuous basis. Patients rested quietly in the supine position for $\geq 30 \mathrm{~min}$ before testing began.

Blood flow and lower limb size change. We used mercury in silastic strain gauge plethysmography to measure forearm blood flow and later calf blood flow while supine. We also measured calf size change and calf blood flow during HUT. Methods were adapted from the work of Gamble et al. $(15,16)$ and are summarized in Figure 1. Occlusion cuffs were first placed around the upper limb approximately $10 \mathrm{~cm}$ above a strain gauge of appropriate size attached to a Whitney-type strain gauge plethysmograph (Hokanson, Inc, Bellevue, WA, U.S.A.). Changes in strain gauge resistance are proportionate to changes in limb area $(A)$. The cuff was inflated suddenly to a pressure of $50 \mathrm{~mm} \mathrm{Hg}$ to prevent venous outflow. Arterial inflow in units of milliliters per $100 \mathrm{~mL}$ of tissue per minute was estimated as the rate of change of the rapid increase in limb volume shown in the bottom panel of Figure 1. This assumes an approximate cylindrical segment of volume, $\pi r^{2} l$, equivalent to $A l$, where $l$ is the length of the cylinder. Thus a small change in limb cross-sectional area gives a change in volume of $l d A$ or a fractional change in volume of $d A / A$, equivalent to a fractional change in area that may be directly obtained from the calibrated plethysmograph output. The rate of change in limb volume with sudden occlusion was estimated by using custom linear least squares analysis using the singular value decomposition algorithm (17). Flow measurements were repeated at least in triplicate. The same procedure was repeated in the calf with the leg congestion cuff placed just above the knee to obtain flows. We estimated forearm and calf $\mathrm{Pv}$ supine by gradually increasing congestion cuff pressure until the first deflection was noted on the strain gauge. (i.e. the limb began to enlarge). This quantity, denoted Pv, has been shown to closely approximate Pv measured during simultaneous invasive monitoring (18). Specific resistance in units of millimeters of mercury-minutes $-100 \mathrm{~mL}$ of tissue per milliliter of flow was estimated by the formula

$$
\mathrm{R}=(\mathrm{MAP}-\mathrm{Pv}) / \text { flow }
$$

where MAP is the mean arterial pressure calculated directly from the time average of the pressure waveform.

Upright testing. POTS patients and control subjects alike were supine for approximately $1 \mathrm{~h}$ in toto before upright tilt testing. After supine vascular measurements were complete, patients were tilted to $70^{\circ}$ for a maximum duration of $30 \mathrm{~min}$ or until syncope, presyncope, or intolerable symptoms occurred. An electrically driven tilt table (Cardiosystems 600, Dallas, TX, U.S.A.) with a footboard for weight bearing was used. The calf strain gauge remained in place throughout HUT and was used to measure the change in calf size with time and 
to measure calf blood flow every $30 \mathrm{~s}$ by intermittent venous occlusion to $70 \mathrm{~mm} \mathrm{Hg}$ (Fig. 1, Top and Middle). The first calf blood flow determination after HUT was obtained within $30 \mathrm{~s}$ of change in position and at $30-\mathrm{s}$ intervals thereafter. Calf blood pressure was determined by oscillometry in the contralateral leg after $5 \mathrm{~min}$ of HUT. Patients with syncope, presyncope, or intolerable symptoms were returned immediately to the supine position and the test terminated. Intractable symptoms included nausea, retching, palpitations, lightheadedness, panic, heat, severe neurocognitive impairment, "blackout," and "whiteout" with or without hypotension. Comparison of flows and size change between POTS patients and control subjects is made difficult by the different durations of tilt. However, in all patients we obtained $\geq 5 \mathrm{~min}$ and often $10 \mathrm{~min}$ of recording.
Statistics. Data were compared by two-way ANOVA. This included time-dependent data. When single measurements were compared and when significant interactions were demonstrated, the ratio of $F$ values was converted to a $t$ distribution using Scheffé's test, and probabilities were thereafter determined. A Bonferroni correction was used to correct for small samples. All results are reported as mean \pm SEM. Statistically significant differences are reported for $p<0.05$.

\section{RESULTS}

Supine data. Supine data are presented in Table 1. Data are presented separately for the subgroup of COI-POTS patients with INOH (INOH group) and the subgroup of COI-POTS patients without INOH (No-INOH group). Data are also pre-

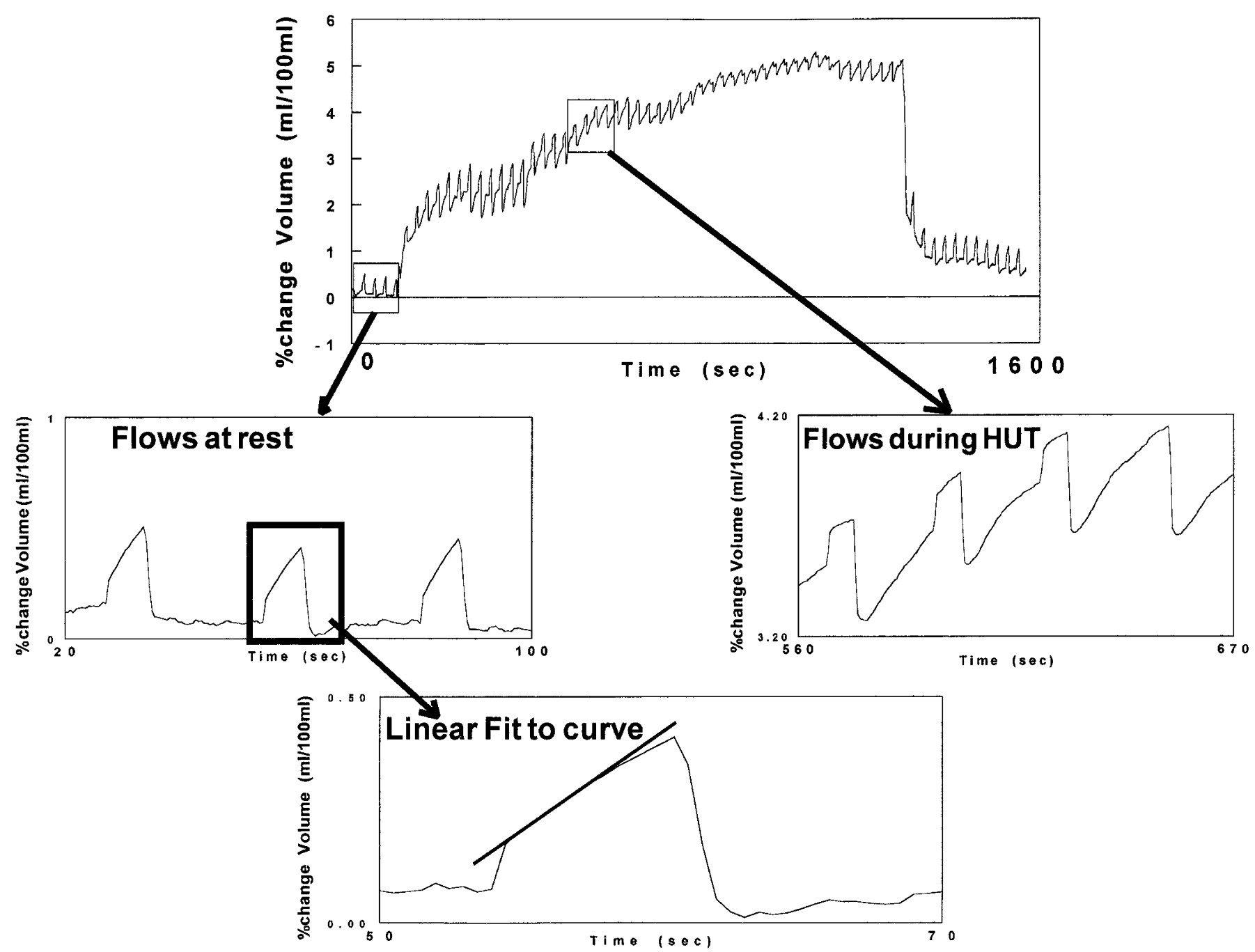

Figure 1. Plethysmographic measurement of flow and diameter. Top, representative measurement of calf flow before and during HUT. During upright tilt, flow measurements are superimposed on a rising baseline because of increased volume of the leg. At least three consecutive baseline measurements of flow are made: Left middle, representative tracings during resting venous occlusion. During tilt, flow is measured every $30 \mathrm{~s}$ while the increasing limb size is continuously assessed. Right middle, upright venous occlusions that are imposed on a rising base caused by an increase in calf size. The rate of change in limb volume with sudden occlusion determines flow and was estimated by using custom linear least squares analysis using the singular value decomposition algorithm. Bottom, single occlusion performed at baseline. Limb volume is shown on the ordinate and is measured in normalized units of milliliters per $100 \mathrm{~mL}$ of tissue or percent change in volume from rest. Thus, a range of $0-6$ refers to a change in calf volume of up to $6 \%$ from resting baseline values. Slope (arterial inflow) as depicted would be measured in normalized units of milliliters per second per $100 \mathrm{~mL}$ of tissue, but data are customarily tabulated in units of milliliters per minute per $100 \mathrm{~mL}$ of tissue by multiplying the measured slope by 60 . 
Table 1. Supine circulatory data

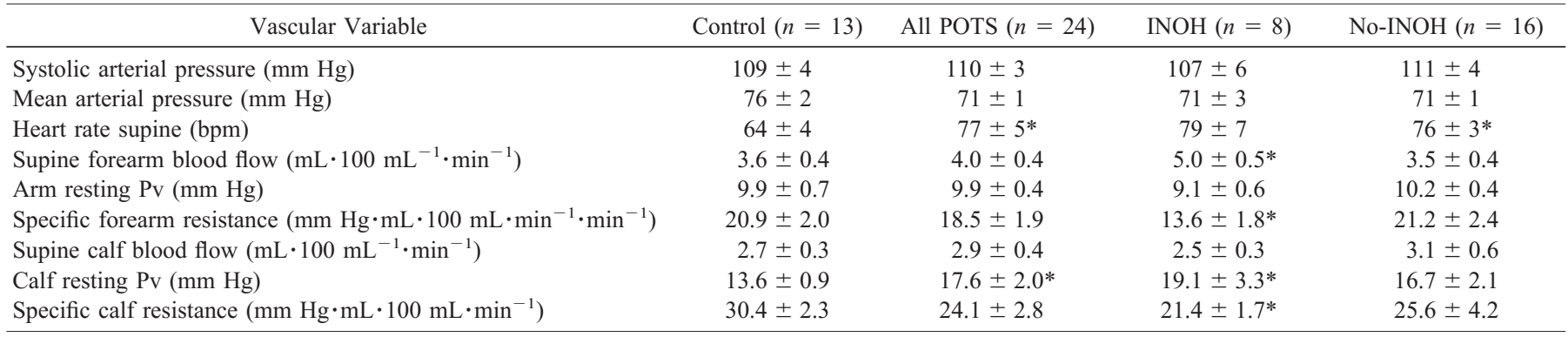

$* p<0.05$ compared with control subjects.

sented for all COI-POTS patients combined. Supine systolic and mean arterial blood pressures were not different from control subjects although heart rate was significantly increased overall in COI-POTS patients and in No-INOH patients. Compared with the control group, the resting supine $\mathrm{Pv}$ was significantly greater overall for COI-POTS and for INOH patients in the calf but not the forearm. Resting forearm blood flow was increased, and forearm and calf resistances were decreased in INOH only.

HUT data. With upright tilt, heart rate increased by $41 \pm 4$ beats per minute in COI-POTS patients and by $16 \pm 5$ beats per minute in control subjects within $10 \mathrm{~min}$ of tilt. All COI-POTS patients developed POTS by definition. Four of 13 control patients suffered vasovagal syncope (abrupt fall of blood pressure and heart rate), which occurred after $13 \mathrm{~min}$ of HUT. Circulatory data presented during the first $10 \mathrm{~min}$ of tilt were not different for control patients that fainted compared with control patients that did not faint.

Circulatory data are summarized in Figures 2 and 3. Figure 2 shows data averaged for all patients and presented as mean \pm SEM for $600 \mathrm{~s}$ of HUT. Blood pressure, calf blood flow, and leg circumference data are represented as percent change from supine rest. This is essential for comparing legs of different size and is natural for comparison of plethysmographic data. As shown in the lower panel of Figure 2, blood pressure declined rapidly in patients with INOH and remained significantly decreased for the duration of tilt. As shown in the middle panel, the drop in blood pressure in INOH was associated with a marked rise in calf blood flow, far exceeding normal values early on and remaining markedly elevated throughout tilt. A similar although less marked trend toward elevated blood flow was noted in the No-INOH group, which reached statistical significance during the first $5 \mathrm{~min}$ of tilt compared with control. For control subjects, after an initial increase, blood flow declined throughout, tilt achieving levels less than baseline $(<1$ on the graph) within a few minutes. Decreased blood pressure and increased flow in COI-POTS patients was associated with a significant increase in calf volume in $\mathrm{INOH}$ patients and a similar although smaller volume increase in the $\mathrm{No}-\mathrm{INOH}$ group.

To further investigate the relation between calf blood flow and instantaneous hypotension, we plotted a scattergram of the maximum fractional increase in blood flow versus minimum blood pressure during early HUT and performed linear regres-
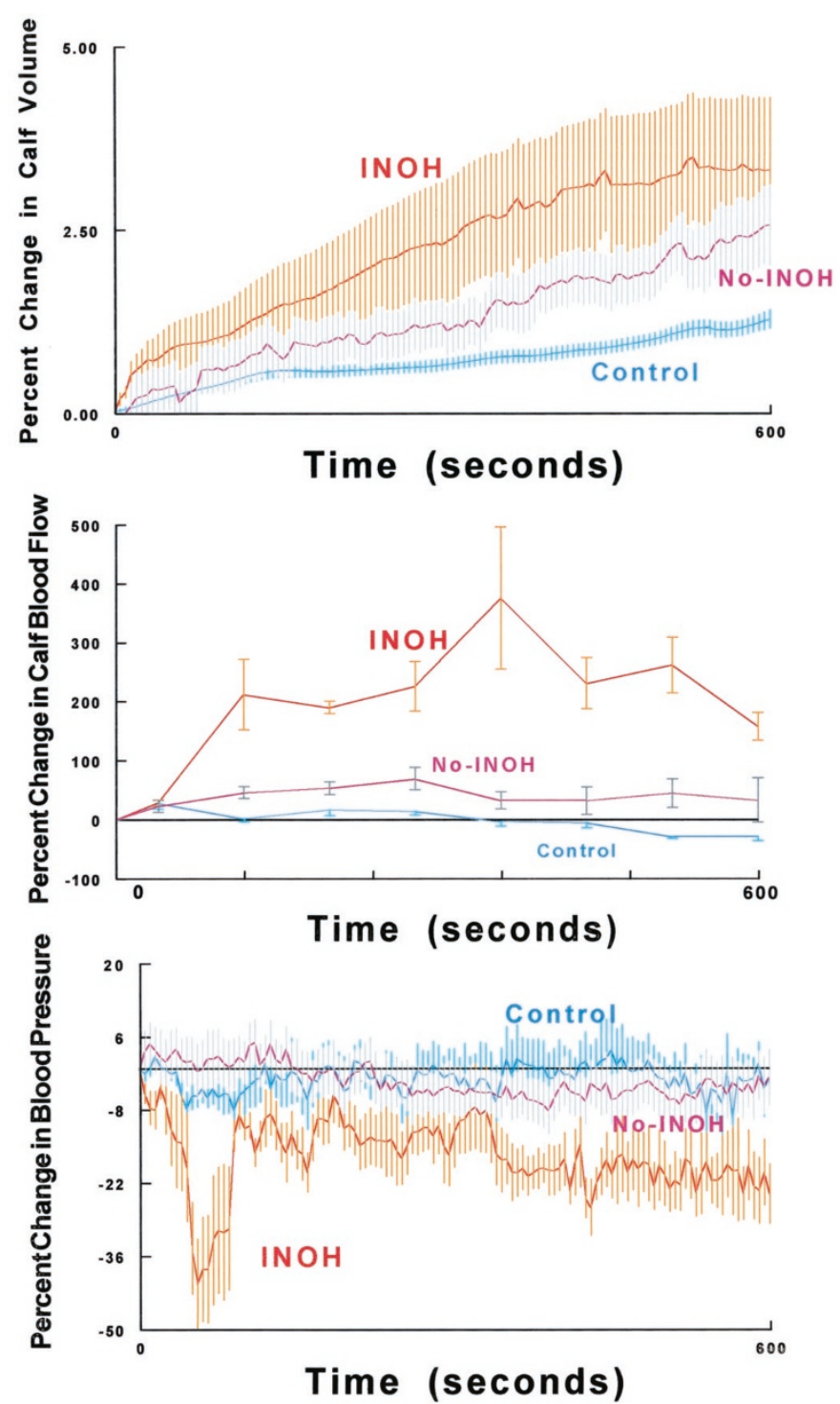

Figure 2. Changes in calf volume, calf blood flow, and arm blood pressure during HUT. Top, percent change (mean \pm SEM) in calf volume during HUT. Volume increases with tilt in all groups but most in INOH patients. Middle, percent change (mean \pm SEM) in calf blood flow from resting conditions during HUT. Blood flow is markedly increased above resting values during the period of observation in INOH patients, modestly elevated above resting in $\mathrm{No}-\mathrm{INOH}$ patients with POTS, and decreased in control because of (appropriate) vasoconstriction. Bottom, percent change (mean \pm SEM) in arm systolic blood pressure from resting control during HUT. Systolic pressure falls immediately after tilt in INOH patients and remains decreased thereafter. 


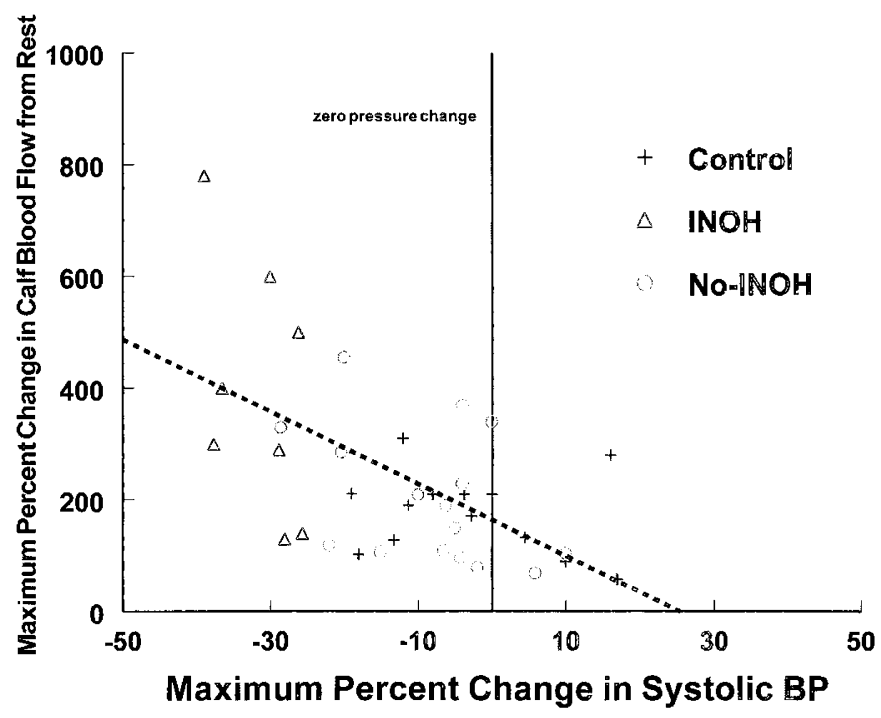

Figure 3. Percent change in systolic blood pressure and flow during HUT. Scattergram relates maximum percent increase in calf blood flow, expressed as percent change of resting flow, to maximum percent change in systolic blood pressure, expressed as a percent change from resting pressure. Coincident maxima occurred soon after the onset of upright tilt. There is a clear negative correlation $(r=-0.72, p<0.001)$. Patients and subjects may be construed as falling on the same straight line.

sion (Fig. 3). Although there is considerable variability, the data suggest a significant inverse relation $(r=-0.72, p<$ 0.001 ) between flow and blood pressure, which may apply to all subjects, control, INOH, and No-INOH alike.

\section{DISCUSSION}

Our data suggest the following:

1. Resting forearm and calf arterial resistance to blood flow is lower than control subjects in COI-POTS patients with INOH.

2. Resting calf $\mathrm{Pv}$ is higher in $\mathrm{INOH}$.

3. With orthostasis, initial calf blood flow increases markedly in patients with INOH, to a lesser degree in COI-POTS patients with No-INOH, and to a small extent in control subjects early during orthostasis.

4. The early increase in calf blood flow is temporally related to early hypotension and enhanced swelling of the lower extremity.

5. Increased blood flow persists during the period of HUT in $\mathrm{INOH}$, remains at or above baseline in No-INOH, and falls steadily in control patients.

6. INOH may be explained as a variant of COI-POTS associated with marked vasoconstrictor deficit of the lower extremities.

Resting supine arterial resistance to blood flow is lower than control subjects in COI-POTS, which implies that vasoconstrictor tone is deficient. On the one hand, these results can be interpreted as being consistent with hypotheses regarding the origins of POTS involving distal extremity denervation, or the so-called "long tract syndrome" $(4,19)$, in which peripheral nerve deficit is confined to the legs. On the other hand, our results suggest a more widespread loss of vasoconstrictor tone involving both arm and leg. However, only an assessment of baseline vasoconstrictor tone (via measurement of peripheral resistance) is made here, whereas further deficits in vasoconstrictor reserve may only become evident when the system is stressed by orthostasis $(20,21)$. We suspect that it is limb dependency rather than an intrinsic difference between leg and arm that is primarily important. Indeed, although during HUT acrocyanosis of the lower extremities are most commonly observed (10), our patients more often complain of coolness, color change, and discomfort in their upper extremities when dependent. Evidence for the denervation hypothesis arises from the work of Streeten (19) and Streeten et al. (22) on "hyperadrenergic orthostatic hypotension," in which persistent orthostatic tachycardia and orthostatic intolerance related to apparent denervation hypersensitivity of the superficial veins of the foot was demonstrated.

Resting calf $\mathrm{Pv}$ is higher in patients with $\mathrm{INOH}$. An arterial constrictor defect as proposed here could account for increased Pv caused by enhanced passive filling of the veins. If there were unchanged venous resistance, one would expect increased supine blood flow, which we did not observe. Therefore it is likely that a change in venous resistance has also occurred in the legs of INOH patients while supine. Altered venous mechanical properties might also explain the findings, but an accurate assessment of venous properties supine and during orthostasis awaits future experimentation. Decreased resistance to blood flow in dependent lower extremities may be related to increased venous filling and enhanced microvascular filtration, resulting in the excessive pooling in POTS patients. These data may also explain the vagal withdrawal and characteristic tachycardia of the syndrome as the result of a peripheral redistribution of fluid causing central hypovolemia (23). Further experiments documenting orthostatic changes in lower extremity blood flow and Pv, venous efflux, and alterations in blood compartmentalization will be necessary to confirm this conjecture.

With orthostasis, initial calf blood flow increases markedly in $\mathrm{INOH}$, to a lesser degree in COI-POTS patients with No$\mathrm{INOH}$, and to a small extent in control subjects early during orthostasis. Arterial vasoconstriction normally occurs during upright tilt resulting in decreased blood flow below resting supine values (24). This is precisely what was observed in our control subjects. Decreased calf flow owing to vasoconstriction evolves with time from HUT, being decreased dependent on gravitational translocation of blood as well as on the rate of sympathetic activation. Because resting resistance is decreased in both arms and legs in COI-POTS patients with INOH, it is not surprising to find enhancement in calf blood flow in these patients compared with control subjects. That blood flow continues to increase in INOH implies persistent vasoconstrictor deficiency in patients with INOH. Persistent vasoconstrictor deficiency reflects a defective efferent baroreflex-mediated vasoconstrictor response. Moreover, the afferent arm of the baroreflex is likely intact because the patients become uniformly tachycardic. No-INOH patients incompletely share such vasoconstrictor deficiency. One might therefore be tempted to hypothesize a continuum of leg vasoconstrictor capability. Such conjecture is supported by our correlative data 
in which calf blood flow is significantly negatively correlated to early blood pressure for all groups, suggesting a similar physiology for early blood flow changes in patients and control subjects alike. Thus, the decrease in blood pressure relates to the rapidity of caudal blood flow. Because only $10 \mathrm{~min}$ of observation is depicted, vascular changes should have little to do with neurohumoral responses, which typically take many more minutes to fully develop (25).

The early increase in calf blood flow is temporally related to early hypotension and enhanced swelling of the lower extremity. This seems clear from Figures 2 and 3. Increased flow increases blood delivery to the dependent limb, increases orthostatic venous pressure, and increases microvascular filtration (26). This is the pooling observed in patients with COIPOTS. The pooled blood and transudate is unavailable for circulation, resulting in central hypovolemia and vagally mediated (23) tachycardia - ergo POTS.

Increased blood flow persists during the period of HUT in INOH patients, remains at or above baseline in $\mathrm{No}-\mathrm{INOH}$ patients, and falls steadily in control patients. Both INOH and No-INOH flow responses are abnormal and encourage pooling changes. The control response is normal and conserves central blood volume. Vasoconstrictor deficiency can explain all findings. Such a global vasoconstrictor defect has also been proposed by the Vanderbilt group in $\mathrm{COI}(7,8)$ and, if persistent, may result in decreased blood volume in some patients with this syndrome associated with increased plasma catecholamines, as noted by Jacob et al. (8). Also, Furlan et al. (7) demonstrated increased muscle sympathetic nerve activity at rest, which did not increase appropriately with orthostatic stress, resulting in blunted sympathetic response to orthostasis, consistent with inappropriate vasoconstriction to orthostatic stress. They found evidence for vagal withdrawal in their patients as the cause for the tachycardic response.

INOH may be explained as a variant of COI-POTS associated with marked vasoconstrictor deficit of the lower extremities. This is arguably an unfair statement. INOH, as introduced by Tanaka and coworkers among children with symptoms of COI, was defined by the response to a rapid standing orthostatic stress test. The normal early response to rapid standing and tilt differ $(27,28)$. Standing, as characterized by the work of Tanaka et al. (29) and Tanaka and Tamai (30), normally causes a brief increase in blood pressure because of the expulsion of blood from the lower extremities and abdominal vasculature during muscular contraction. This is followed by a rapid fall in blood pressure, owing primarily to gravity causing nearly instantaneous activation of the vagal arm of the baroreflex producing tachycardia, and slower baroreflex-mediated vasoconstriction producing a brief overshoot in blood pressure, which decreases toward control levels (albeit with increased diastolic pressure) while heart rate declines to levels elevated somewhat above baseline. Similar findings are not found in tilts performed in normal subjects (28). The tilting process is performed over several seconds (typically $8-10 \mathrm{~s}$ ), ideally with minimal contraction of the legs or abdominal muscles. Systolic blood pressure tends to increase slightly, although modest transient decreases are often initially observed. Diastolic blood pressure increases. Heart rate increases. Within 1-2 min the hemodynamic responses during HUT approximate those during active standing.

Therefore, it is not entirely appropriate to compare HUT results in patients with COI with standing results in patients with COI. For example, decreased blood pressure in our $\mathrm{INOH}$ patients were rarely $>40 \mathrm{~mm} \mathrm{Hg}$ whereas the mean fall in systolic blood pressure in INOH patients in the study by Tanaka et al. (1) was $55 \mathrm{~mm} \mathrm{Hg}$. Nevertheless, we strongly suspect that those INOH patients are physiologically indistinguishable from our INOH patients. It is unfortunate that our current instrumentation precludes accurate data collection during rapid standing. Moreover, our data, as well as those of Tanaka et al., $(1,2)$, suggest a mechanism for INOH that is dependent on defective vasoconstriction. Our data complement and supplement those of Tanaka et al. (1) by implying that INOH is a variant of POTS in which vasoconstriction is impaired even at rest, and that correlative analysis suggest that vasoconstrictive capabilities form a continuum stretching from normal subjects to patients with varying early changes in blood pressure in response to orthostatic stress.

Acknowledgments. The authors thank Drs. Gary Wormser and Michael Gewitz for their support and critical advice.

\section{REFERENCES}

1. Tanaka H, Yamaguchi H, Matushima R, Ninomiya H, Ozaki T, Tamai H 1999 Instantaneous orthostatic hypotension in children and adolescents: a new entity of orthostatic intolerance. Pediatr Res 46:691-696

2. Tanaka H, Yamaguchi H, Kim Y, Mino M, Takenaka Y, Konishi K 1993 Instantaneous reduction of blood pressure observed on standing up in children with psychosomatic symptoms. Acta Paediatr Jpn 97:941-946

3. MacLean AR, Allen EV 1940 Orthostatic hypotension and orthostatic tachycardia: treatment with the "head-up" bed. JAMA 115:2162-167

4. Schondorf R, Low PA 1993 Idiopathic postural orthostatic tachycardia syndrome: an attenuated form of acute pandysautonomia? Neurology 43:132-137

5. Low PA, Novak V, Novak P, Sandroni P 1997 Postural tachycardia syndrome. In: Low PA (ed) Clinical autonomic disorders, 2nd Ed. Lippincott-Raven, Philadelphia, pp 681-699

6. Grubb BP, Kosinski DJ, Boehm K, Kip K 1997 Postural orthostatic tachycardia syndrome: a neurocardiogenic variant identified during head-up tilt table testing. Pacing Clin Electrophysiol 20:2205-2212

7. Furlan R, Jacob G, Snell M, Robertson D, Harris P, Mosqueda-Garcia R 1998 Chronic orthostatic intolerance: a disorder with discordant cardiac and vascular sympathetic control. Circulation 98:2154-2159

8. Jacob G, Shannon JR, Black B, Biaggoni I, Mosqueda-Garcia R, Robertson RM, Robertson D 1997 Effects of volume loading and pressor agents in idiopathic orthostatic tachycardia. Circulation 96:575-580

9. Stewart JM, Weldon A, Munoz J 1999 Patterns of orthostatic intolerance: the orthostatic tachycardia syndrome and adolescent chronic fatigue. J Pediatr 135:218225

10. Stewart JM, Gewitz MH, Weldon A, Arlievsky A, Li K, Munoz JL 1999 The nature of orthostatic intolerance in adolescents with the chronic fatigue syndrome. Pediatrics 103:116-121

11. Karas B, Grubb BP, Boehm K, Kip K 2000 The postural orthostatic tachycardia syndrome: a potentially treatable cause of chronic fatigue, exercise intolerance, and cognitive impairment in adolescents. Pacing Clin Electrophysiol 23:344-351

12. Rowe PC, Bou-Holaigh I, Kan JS, Calkins H 1995 Is neurally mediated hypotension an unrecognized cause of chronic fatigue? Lancet 345:623-624

13. Fukuda K, Straus SE, Hickie I, Sharpe MC, Dobbins JG, Komaroff A 1994 The chronic fatigue syndrome: a comprehensive approach to its definition and study. Ann Intern Med 121:953-959

14. Bannister R, Schatz I 1996 Consensus statement on the definition of orthostatic hypotension, pure autonomic failure, and multiple system atrophy. Clin Auton Res 6:125-126

15. Gamble J, Gartside IB, Christ F 1993 A reassessment of mercury in silastic strain gauge plethysmography for microvascular permeability assessment in man. J Physiol (Lond) 464:407-422

16. Gamble J, Christ F, Gartside IB 1997 The effect of passive tilting on microvascular parameters in the human calf: a strain gauge plethysmography study. J Physiol (Lond) 498:541-552

17. Press WH, Teukolsky SA, Vetterling WT, Flannery BP 1992 Numerical recipes in C, 2nd Ed. Cambridge University Press, Cambridge, UK, pp 59-70 
18. Christ F, Gamble J, Baschnegger H, Gartside IB 1997 Relationship between venous pressure and tissue volume during venous congestion plethysmography in man. J Physiol (Lond) 503:463-467

19. Streeten DP 1990 Pathogenesis of hyperadrenergic orthostatic hypotension: evidence of disordered venous innervation exclusively in the lower limbs. J Clin Invest $86: 1582-1588$

20. Brown CM, Hainsworth R 2000 Forearm vascular responses during orthostatic stress in control subjects and patients with posturally related syncope. Clin Auton Res 10:57-6

21. Schondorf R, Wieling W 2000 Vasoconstrictor reserve in neurally mediated syncope. Clin Auton Res 10:53-55

22. Streeten DP, Anderson G, Richardson R, Thomas FD 1988 Abnormal orthostatic changes in blood pressure and heart rate in subjects with intact sympathetic nervous function: evidence for excessive venous pooling. J Lab Clin Med 111:326-335

23. Stewart JM 2000 Autonomic nervous system dysfunction in adolescents with postural orthostatic tachycardia syndrome and the chronic fatigue syndrome is characterized by attenuated vagal baroreflex and potentiated sympathetic vasomotion. Pediatr Res 48:218-226

24. Bridgen W, Howarth S, Sharpey-Schafer EP 1950 Postural changes in the peripheral blood flow of normal subjects with observations of vasovagal fainting reactions, as a result of tilting, the lordotic posture, pregnancy and spinal anesthesia. Clin Sci 9:79-91

25. Mohanty PK, Sowers JR, Beck FWJ, Thames MD 1988 Reflex effects of prolonged cardiopulmonary baroreceptor unloading in humans. Am J Physiol 254:R320-R324

26. Brown CM, Hainsworth R 1999 Assessment of capillary fluid shifts during orthostatic stress in normal subjects and subjects with orthostatic intolerance. Clin Auton Res 9:69-73

27. Sprangers RL, Wesseling KH, Imholz AL, Imholz BP, Wieling W 1991 Initial blood pressure fall on stand up and exercise explained by changes in total peripheral resistance. J Appl Physiol 70:523-530

28. Sprangers RL, Veerman DP, Karemaker JM, Wieling W 1991 Initial circulatory responses to changes in posture: influence of the angle and speed of tilt. Clin Physiol 11:211-220

29. Tanaka H, Thulesius O, Borres M, Yamaguchi H, Mino M 1994 Blood pressure responses in Japanese and Swedish children in the supine and standing position. Eur Heart J 15:1011-1019

30. Tanaka H, Tamai H 1998 Recent advances in autonomic function tests of the cardiovascular system in children. Med Princ Pract 7:157-171 